\title{
Hydrogen Sulfide Provides Intestinal Protection During A Murine Model of Experimental Necrotizing Enterocolitis
}

\author{
Natalie A. Drucker $\mathrm{MD}^{1,3}$, Amanda R. Jensen $\mathrm{MD}^{1,3}$, Michael Ferkowicz $\mathrm{PhD}^{1,3}$, \\ and Troy A. Markel MD ${ }^{1,2,3}$ \\ ${ }^{1}$ Department of Surgery, Section of Pediatric Surgery \\ ${ }^{2}$ Riley Hospital for Children at Indiana University Health and \\ ${ }^{3}$ The Indiana University School of Medicine \\ Indianapolis, IN
}

NAD performed animal care and experiments and drafted the manuscript, NAD and ARJ performed histological grading and statistical analysis, NAD performed protein isolation and tissue analysis, MJF prepared histology slides, TAM contributed critical ideas, assistance and manuscript advice. All authors provided critical revisions to the manuscript and assisted with its final preparation.

No disclosures to report.

\section{$\underline{\text { KEY WORDS }}$}

animal model, necrotizing enterocolitis, hydrogen sulfide, premature, neonatal, intestine, ischemia

This work was made possible with support from:

1) KL2TR001106 (A. Shekhar, $\mathrm{Pl}$ ) from the National Institutes of Health, National Center for Advancing Translational Sciences, Clinical and Translational Sciences Award

2) Indiana University Health, Indianapolis, IN

\section{Correspondence:}

Troy A. Markel, MD

Assistant Professor of Surgery

Indiana University School of Medicine

Riley Hospital for Children at IU Health

705 Riley Hospital Dr.

RI 2500

Indianapolis, IN 46202

Phone: 317-437-2506

Fax: 317-274-4491 


\section{ABSTRACT:}

Background: Necrotizing enterocolitis (NEC) continues to be a morbid surgical condition among preterm infants. Novel therapies for this condition are desperately needed. Hydrogen sulfide $\left(\mathrm{H}_{2} \mathrm{~S}\right)$ is an endogenous gasotransmitter that has been found to have beneficial properties. We therefore hypothesized that intraperitoneal injection of various $\mathrm{H}_{2} \mathrm{~S}$ donors would improve clinical outcomes, increase intestinal perfusion, and reduce intestinal injury in an experimental mouse model of necrotizing enterocolitis.

Methods: NEC was induced in five-day-old mouse C57BL/6 mouse pups through maternal separation, formula feeding, and intermittent hypoxic and hypothermic stress. The control group $(n=10)$ remained with their mother and breastfed ad lib. Experimental groups ( $n=10$ / group) received intraperitoneal injections of phosphate buffered saline (PBS) vehicle or one of the following $\mathrm{H}_{2} \mathrm{~S}$ donors: (1) GYY4137, $50 \mathrm{mg} / \mathrm{kg}$ daily; (2) Sodium sulfide ( $\left.\mathrm{Na}_{2} \mathrm{~S}\right), 20 \mathrm{mg} / \mathrm{kg}$ three times daily; (3) AP39, $0.16 \mathrm{mg} / \mathrm{kg}$ daily. Pups were monitored for weight gain, clinical status, and intestinal perfusion via transcutaneous Laser Doppler Imaging (LDI). After sacrifice on day nine, intestinal appearance and histology were scored and cytokines were measured in tissue homogenates of intestine, liver, and lung. Data were compared with Mann-Whitney and $\mathrm{p}<0.05$ was considered significant.

Results: Clinical score and weight gain were significantly improved in all three $\mathrm{H}_{2} \mathrm{~S}$ treated groups as compared to vehicle ( $p<0.05$ for all groups). Intestinal perfusion of the vehicle group was $22 \%$ of baseline while the GYY4137 group was $38.7 \%$ 
( $p=0.0103), \mathrm{Na}_{2} \mathrm{~S}$ was $47.0 \%(p=0.0040)$, and AP39 was $43.0 \%(p=0.0018)$. The vehicle group had a median histology score of 2.5, while the GYY4137 group's was 1 $(p=0.0013), \mathrm{Na}_{2} \mathrm{~S}$ was $0.5(p=0.0004)$, and AP39 was $0.5(p=0.0001)$. Cytokine analysis of the intestine of the $\mathrm{H}_{2} \mathrm{~S}$-treated groups revealed levels closer to breastfed pups as compared to vehicle ( $p<0.05$ for all groups).

Conclusion: Intraperitoneal administration of $\mathrm{H}_{2} \mathrm{~S}$ protects against development of NEC by improving mesenteric perfusion, and by limiting mucosal injury and altering the tissue inflammatory response. Further experimentation is necessary to elucidate downstream mechanisms prior to clinical implementation. 


\section{INTRODUCTION:}

Necrotizing enterocolitis (NEC) is the leading cause of morbidity and mortality from gastrointestinal disease in the premature neonatal population. Incidence has been estimated to vary from $12 \%$ to $20 \%$ of preterm infants, with mortality as high as $40 \%$ of those affected [1-4]. Despite NEC being the most common acquired gastrointestinal emergency among neonates, relatively little advancement has been made in available medical therapies over the last few decades. Surgical resection of necrotic bowel is required in up to $50 \%$ of cases [5]. Morbidities are significant, including intestinal stricture and short bowel syndrome as well as growth retardation and neurodevelopmental delay [3]. Therefore, a novel medical therapy for this condition is desperately needed.

One recently appreciated therapy for ischemic tissue injury has been hydrogen sulfide gas $\left(\mathrm{H}_{2} \mathrm{~S}\right)$. Recently lauded alongside nitric oxide and carbon monoxide as one of the three major gasotransmitters, it has been found to have anti-apoptotic, antioxidant, anti-inflammatory, and vasodilatory properties [6]. Though many cells produce it endogenously, it can also be applied exogenously with similar effects. In animal models, $\mathrm{H}_{2} \mathrm{~S}$ administration has been shown to provide protection against ischemic injury in the myocardium and brain [4, 7-9]. In mouse studies of intestinal ischemia and reperfusion, $\mathrm{H}_{2} \mathrm{~S}$ was applied directly to the ischemic bowel resulting in improved intestinal perfusion and decreased histologic injury secondary to the insult [4]. Additionally, Li et all has demonstrated in vitro protection in intestinal epithelial cells [10]

$\mathrm{H}_{2} \mathrm{~S}$ exists in its native form as a gas, but various substances act as $\mathrm{H}_{2} \mathrm{~S}$ donors in aqueous solution. These can be classified further into inorganic sulfide salts and 
synthetic molecules. Sodium hydrosulfide ( $\mathrm{NaHS})$ and sodium sulfide $\left(\mathrm{Na}_{2} \mathrm{~S}\right)$ are the two most commonly used sulfide salts, and are often sold in their hydrated forms. Two frequently used synthetic molecules are GYY4137 (morpholin-4-ium 4methoxyphenyl(morpholino) phosphinodithioate) and AP39 ((10-oxo-10-(4-(3-thioxo-3H1,2-dithiol-5yl)phenoxy)decyl) triphenylphosphonium bromide) [11]. These are both relatively large molecules that produce hydrogen sulfide at a consistent rate over time, leading to stable concentrations in aqueous solution $[12,13]$. It is thought that AP39 acts specifically on the mitochondria, while GYY4137 is more generalized [14]. All of these donors have been used in animal experiments without prohibitive adverse effects $[12,13,15]$

NEC is characterized not only by intestinal injury, but also dyscrasias in local and systemic inflammatory cytokine cascades. Interleukin 6 (IL-6), typically thought of as an acute phase reactant, has both inflammatory and anti-inflammatory properties, and may act in a protective manner against intestinal injury $[16,17]$. Interleukin $1 \beta(\mathrm{IL}-1 \beta)$, another acute phase reactant, is produced by many cell types, including the endothelium, in response to stress [18]. Meanwhile, a variety of angiogenic growth factors, such as vascular endothelial growth factor (VEGF), are released by the endothelium to circumvent hypoxic injury [19]. Additionally, interleukin 12 (IL-12) released by the mucosa is thought to be directly related to bacterial clearance and mucosal permeability and therefore is an important marker in experimental NEC [20]. These four signaling molecules are some of the many that are affected in experimental NEC. 
Development of NEC is certainly multifactorial, but one contributor seems to be intestinal ischemia and necrosis [3]. Because of this, it is plausible that $\mathrm{H}_{2} \mathrm{~S}$ donors would be beneficial for the treatment of NEC. We therefore hypothesized that intraperitoneal injection of $\mathrm{H}_{2} \mathrm{~S}$ donors would improve clinical outcomes, increase intestinal perfusion, and reduce intestinal injury in an experimental mouse model of necrotizing enterocolitis.

\section{MATERIALS AND METHODS}

\section{Experimental NEC Model:}

Indiana University Institutional Animal Care and Use Committee approved the experimental protocol and animal use. We used a previously well published and validated model of experimental NEC [21, 22]. C57BL/6 mouse pups (bred in house from adult mice obtained from Jackson Labs, Bar Harbor, ME) were permanently separated from their mother on postnatal day $(P)$ five. They were housed in a neonatal incubator from P5 to P9 with humidity $40 \%$ and temperature $32^{\circ} \mathrm{C}$. The control group $(n=10)$ remained with their mother and breastfeed ad libitum. Experimental groups ( $n=10$ per group) were gavage fed with a 2 French catheter three times daily with hyperosmolar formula. Formula was prepared using $4 \mathrm{~g}$ of Esbilac canine supplement and $6 \mathrm{~g}$ Similac in $20 \mathrm{~mL}$ of nanopure filtered water (Barnstead Nanopure, APS Water Services Inc., Van Nuys, CA). Formula was discarded and prepared fresh every 48 hours. Animals were fed $300 \mathrm{kcal} / \mathrm{kg} /$ day and all feeds were supplemented with 8 $\mathrm{mg} / \mathrm{kg}$ lipopolysaccharide (lipopolysaccharides from Escherichia coli O111:B4, SigmaAldrich Company LLC, Dorset, UK). Before each feed, pups were placed in a chamber 
with $5 \% \mathrm{O}_{2}$ and $95 \% \mathrm{~N}_{2}$ for 10 minutes. After the morning and evening feed, pups were placed in the $4^{\circ} \mathrm{C}$ refrigerator for 10 minutes. Mice who died less than 24 hours into the protocol were excluded, as their death was more likely due to causes other than NEC. Any animal whose tissue was liquefied or unusable for histologic evaluation (i.e. died overnight and discovered hours later) was excluded completely from evaluation. Other animals that died during the study but were identified immediately were still included in analysis for all data points.

\section{$\underline{H_{2}} \underline{S}$-donor Administration:}

Before hypoxic stress, experimental groups received intraperitoneal injections of phosphate buffered saline (PBS) vehicle or one of the following $\mathrm{H}_{2} \mathrm{~S}$ donors in PBS solution: (1) GYY4137, $50 \mathrm{mg} / \mathrm{kg}$ daily; (2) $\mathrm{Na}_{2} \mathrm{~S}, 20 \mathrm{mg} / \mathrm{kg}$ three times daily; (3) AP39, $0.160 \mathrm{mg} / \mathrm{kg}$ daily. $\mathrm{Na}_{2} \mathrm{~S}$ was dosed more frequently because as a salt in aqueous solution, its $\mathrm{H}_{2} \mathrm{~S}$ production is known to be short-lived, while the synthetic donors produce at least 24 hours of a stable $\mathrm{H}_{2} \mathrm{~S}$ concentration in aqueous solution [12, 23]. Total volume of each injection was $10 \mu \mathrm{L}$. Control (breastfed) groups did not receive any intraperitoneal injections. Doses are based on previous animal model studies of cardiac ischemia and reperfusion [15].

\section{Clinical Assessment:}

Control groups were assessed daily, while experimental groups were assessed with each feed. Pups were assessed in a systematic fashion to ensure consistency. 
The clinical sickness score was previously published by Zani [21]. The reported clinical assessment score was the pup's last score prior to death or euthanasia (Table 1).

\section{Perfusion Analysis:}

Intestinal perfusion was analyzed transcutaneously with a Laser Doppler Perfusion Imager (LDI; Moor Instruments, Wilmington, DE). As the laser was known to penetrate skin up to several millimeters thick, our previously published protocols were modified to allow for transcutaneous acquisition in the neonatal pups in order to avoid multiple surgeries [4]. Pups were held supine by their front limbs and tail and images were acquired of the entire torso and abdomen. Using the computer software, a region of interest was created from the diaphragm to the pelvis to obtain a flux mean perfusion value within this region. Full, visible bladders were excluded from the region of interest for consistency. Perfusion was expressed as a percentage of baseline perfusion on P5. Pups that died during the protocol ( 1 in vehicle group, 1 in $\mathrm{Na}_{2} \mathrm{~S}$ group, and 1 in AP39 group) were included in evaluation with a perfusion value of $0 \%$.

\section{Macroscopic Gut Assessment and Histology:}

On P9, pups were sacrificed by decapitation and intestinal appearance was assessed with a macroscopic scoring system based on color, consistency, and dilation of the intestine as described by Zani et. al. (Table 2). The terminal ileum and distal jejunum was then formalin fixed, paraffin embedded, and stained with hematoxylin and eosin. Two blinded authors evaluated degree of injury by histology. The histological scoring system used was published by Zani et. al [21] with scores ranging from 0 to 4 . 
$0=$ normal intestine $; 1=$ some disarrangement of villus enterocytes, villus-core separation; 2 = significant disarrangement of villus enterocytes, villus-core separation down sides of villi, blunting of villi; $3=$ epithelial sloughing of villi, loss of villi; $4=$ necrosis. A score of 2 or higher indicated presence of NEC, with 3 or higher indicating severe NEC.

Intestinal Cytokine Analysis:

Murine organs were acquired and processed for proteins as previously described $[4,24,25]$. Following euthanasia, part of the small intestine, liver, and lung was snap frozen in liquid nitrogen and stored at $-80^{\circ} \mathrm{C}$. Tissue was thawed and homogenized with the Bullet Blender (Next Advance, Averill Park, NY) in RIPA buffer (Sigma, St. Louis, MO) with 1:100 dilutions of both phosphatase and protease inhibitors (Sigma, St. Louis, MO). After homogenization, samples were centrifuged at 12,000 rpm and supernatants were collected for further analysis. Total protein was quantified with the Bradford Assay using a spectrophotometer (VersaMax microplate reader, Molecular Devices, Sunnyvale, CA).

Murine interleukin 6 (IL-6), vascular endothelial growth factor (VEGF), interleukin $1 \beta$ (IL-1ß), and interleukin 12 (IL-12) were measured using ELISA (R\&D Systems, BioTechne Corporation, Minneapolis, MN). ELISAs were repeated to ensure consistency and values were normalized to the control group. Assays were performed at 1:20 dilution and cytokine concentrations were normalized to total protein for each sample.

\section{Statistical Analysis:}


Ordinal data was reported using median and interquartile range. Continuous variables were reported as mean \pm SEM. All non-parametric data was compared using the Mann-Whitney U test. GraphPad Prism 7 (GraphPad Software, La Jolla, CA) was used for all statistical analysis and figures. $P$ values less than 0.05 were considered statistically significant.

\section{RESULTS}

$\underline{H_{2}} \underline{S}$ improves weight gain and clinical status in experimental NEC.

The breastfed group gained an average of $2.23 \pm 0.10$ grams over 4 days, while the PBS-treated NEC group averaged a gain of $0.01 \pm 0.02$ grams $(p<0.0001)$. Although the overall weight gain was minimal, compared to the vehicle treated NEC group, each $\mathrm{H}_{2} \mathrm{~S}$-treated NEC group gained significantly more weight. The GYY4137 group gained $0.16 \pm 0.03$ grams $(p=0.0007)$, the $\mathrm{Na}_{2} \mathrm{~S}$ group gained $0.15 \pm 0.05$ grams $(p=0.0142)$, and the AP39 group gained $0.09 \pm 0.02$ grams $(p=0.0146$, Figure $1 A)$

Clinical assessment scores were significantly worse in the vehicle NEC group compared to the breastfed animals and improved in the $\mathrm{H}_{2} \mathrm{~S}$-treated groups (Figure 1B). While the median score in the control group was $0(I Q R=0-0)$, that of the vehicle NEC group was 3 (IQR=1.75-5.25, $\mathrm{p}=0.0001)$. The median score for the $\mathrm{H}_{2} \mathrm{~S}$-treated NEC groups were lower: GYY4137: 1 (IQR=0-2, p=0.0109), $\mathrm{Na}_{2} \mathrm{~S}: 1$ (IQR=0-1, p=0.0027), and AP39: 1 (IQR=1-2, $\mathrm{p}=0.0185)$.

\section{$\underline{H}_{2}$ S improves intestinal perfusion in experimental NEC.}


At P9, the mean perfusion of the breastfed group was $54.35 \% \pm 5.28 \%$ of baseline on P5. The vehicle group's P9 perfusion was $24.16 \% \pm 4.45 \%$ which was significantly lower than the control group $(\mathrm{p}=0.0015)$. The $\mathrm{H}_{2} \mathrm{~S}$-treated NEC groups had higher perfusion than the vehicle NEC group (Figure 2). At P9, perfusion of the GYY4137 group was $38.69 \% \pm 2.03 \%(p=0.0079), \mathrm{Na}_{2} \mathrm{~S}$ was $46.95 \% \pm 7.93 \%$ ( $p=0.0162)$, and the AP39 group was $37.48 \% \pm 5.24 \%(p=0.0296)$.

\section{$\underline{H}_{2} \underline{S}$ reduces intestinal injury in experimental NEC.}

Median macroscopic injury score of the breastfed group was 0 (IQR=0-0) and that of the vehicle treated NEC group was $2.5(\mathrm{IQR}=2-3, \mathrm{p}<0.0001)$. The median score of the GYY4137 group was 1 (IQR=0-1, $\mathrm{p}<0.0001)$, the $\mathrm{Na}_{2} \mathrm{~S}$ group was 0.5 (IQR=0-1, $p<0.0001)$ and the AP39 group was $1(I Q R=0-2.25, p=0.0103$, Figure $3 A)$. Histologic injury followed a similar pattern with a median score of $0.5(\mathrm{IQR}=0-1)$ for the breastfed pups and $2.5(\mathrm{IQR}=1.5-3)$ for vehicle-treated NEC pups $(\mathrm{p}<0.0001)$. All $3 \mathrm{H}_{2} \mathrm{~S}$-treated groups had improved histology compared to vehicle. The GYY4137 group's median score was $1(\mathrm{IQR}=1-1.625, \mathrm{p}=0.0011)$, the $\mathrm{Na}_{2} \mathrm{~S}$ group's was 0.5 (IQR=0.5-0.125, $\mathrm{p}=0.0004)$, and the AP39 group's was $0.5(\mathrm{IQR}=0.5-1.125, \mathrm{p}=0.0001)$. Incidence of NEC in the Vehicle group was $70 \%$, and it was $20 \%, 10 \%$, and $0 \%$ in the GYY4137, $\mathrm{Na}_{2} \mathrm{~S}$, and AP39 groups respectively. Severe NEC was present in $30 \%$ of the vehicletreated animals, and $0 \%$ of the $\mathrm{H}_{2} \mathrm{~S}$-treated animals (Figure 3B).

$\underline{H}_{2} \underline{S}$ improves local inflammation in the intestine during experimental NEC. 
Intestinal cytokines and growth factors were altered in the experimental NEC model. Animals in the vehicle group had significant derangements in intestinal IL-6, VEGF, IL-1 $\beta$ and IL-12 compared to breastfed control (Figure 4). IL-6, IL-1 $\beta$ and IL-12 were all significantly decreased, while VEGF was increased in the vehicle treated NEC group. Treatment with each of the $\mathrm{H}_{2} \mathrm{~S}$ donors brought these cytokines back toward control levels.

\section{$\underline{H}_{2} \underline{S}$ improves systemic inflammation during experimental NEC.}

Liver and lung cytokines and growth factors were also affected in the experimental NEC model with significant deviations in the vehicle group as compared to control (Figure 5). In both lung and liver, IL-6, VEGF, and IL-1 $\beta$ were significantly reduced in the vehicle treated NEC group compared to control. Only treatment with GYY4137 resulted in statistically significant improvements in IL-6 and VEGF in both the lung and liver.

\section{DISCUSSION}

Necrotizing enterocolitis is a devastating condition of the premature neonate that often requires extensive surgical resection of affected intestine for adequate treatment. Consequences of this disease result in high morbidity and mortality rates across this population. A medical therapy that would reduce the need for surgical treatment is certainly needed. Herein, we demonstrated that treatment with hydrogen sulfide (GYY4137, $\mathrm{Na}_{2} \mathrm{~S}$, and AP39) can provide protection in a mouse model of experimental 
NEC by improving mesenteric perfusion, reducing mucosal injury, and by normalizing local and systemic cytokine and growth factor production.

$\mathrm{H}_{2} \mathrm{~S}$ has recently been appreciated as a gas mediator like carbon monoxide and nitric oxide. Though it is synthesized endogenously from the amino acid cysteine, it can also be administered exogenously via an $\mathrm{H}_{2} \mathrm{~S}$ donor. $\mathrm{H}_{2} \mathrm{~S}$ donor administration has been shown to be beneficial in experimental models of ischemic injury $[4,6,15,26,27]$. Because NEC is mediated, at least in part, through intestinal ischemia and necrosis, $\mathrm{H}_{2} \mathrm{~S}$ therapy may be of benefit in this situation as well.

We noted an improvement in overall clinical status, including weight gain, in mice treated with $\mathrm{H}_{2} \mathrm{~S}$. It is likely that these animals are not only healthier due to decreased systemic inflammation, but also may absorb nutrients more efficiently due to less diseased bowel. It has previously been postulated that local and systemic inflammation during NEC is at least partly responsible for the known sequelae of weight loss and clinical deterioration in neonates [28].

Improvement in intestinal perfusion was another observed benefit of $\mathrm{H}_{2} \mathrm{~S}$ donor administration. $\mathrm{H}_{2} \mathrm{~S}$ is thought to modulate intestinal blood flow, in part as a direct intracellular messenger, but additionally through nitric oxide (NO) mediated effects [4]. Both $\mathrm{H}_{2} \mathrm{~S}$ and $\mathrm{NO}$ are potent smooth muscle relaxants, likely leading to vessel dilatation and improved mesenteric perfusion [29]. One of the possible mechanisms for this interaction involves $\mathrm{H}_{2} \mathrm{~S}$ mediated upregulation of endothelial nitric oxide synthase (eNOS). $\mathrm{H}_{2} \mathrm{~S}$ has been shown to sulfhydrate critical cysteine residues on eNOS, thereby causing enzyme dimerization and upregulation of NO production [30]. Other 
studies have demonstrated that eNOS is an integral intermediary in hydrogen sulfide's ability to improve intestinal perfusion [4].

Bowel injury was also reduced both macroscopically and histologically compared to vehicle control. While this is surely due in part to improved mesenteric perfusion, other beneficial actions of $\mathrm{H}_{2} \mathrm{~S}$ may be at play as well. Other targets of protein sulfhydration and modification include potassium channels, phosphatases, transcriptions factors, and other integral cell-signaling pathways [31, 32]. Sulfhydration can also directly protect proteins and preserve function by preventing oxidation at cysteine residues [33]. Additionally $\mathrm{H}_{2} \mathrm{~S}$ has anti-apoptotic and anti-inflammatory properties, mediated through the NF-KB pathways [34]. These combined effects may have a direct effect on the intestinal epithelium outside of its effects on the mesenteric vasculature.

The experimental NEC model also led to significant derangements in cytokines and growth factors in the intestine. IL-6 was decreased in our NEC model, but returned to near breastfed control levels after the application of $\mathrm{H}_{2} \mathrm{~S}$ donors. Though IL-6 is usually thought of as pro-inflammatory, some studies have suggested that it plays a more protective role in the intestine and acts as an anti-inflammatory mediator. This effect is likely mediated through its influence on other cytokines such as IL-1 [35]. One additional possibility is that by the time the tissue is harvested, the IL-6 has been exhausted from severe inflammation over multiple days. Further investigation into IL-6's role in intestinal ischemic injury is needed.

VEGF is produced by endothelial cells in response to hypoxia or stress [18]. It was elevated in the vehicle group, but reduced by treatment with $\mathrm{H}_{2} \mathrm{~S}$ donors. This 
would suggest that the endothelium was less stressed after administration of hydrogen sulfide donors. VEGF is also considered a growth factor and it plays a role in regulation of angiogenesis [19]. The results of this study might suggest that a higher level of angiogenesis was not necessary following $\mathrm{H}_{2} \mathrm{~S}$ administration.

IL-1 $\beta$ was consistently decreased in our NEC model, and returned to near normal levels with $\mathrm{H}_{2} \mathrm{~S}$ treatment. IL-1 $\beta$ is a secretory molecule that is released from macrophages. It is typically pro-inflammatory, however, has not been shown to be elevated in NEC in previous studies [36]. It also plays a role in gut integrity and enteric mucosal protection by stimulating glial cells [37]. It therefore stands to reason that it may be reduced in experimental NEC, when the intestine is injured and gut barrier permeability is higher than baseline.

IL-12 was decreased in the vehicle group, and increased toward baseline with $\mathrm{H}_{2} \mathrm{~S}$ administration. IL-12 helps to promote the actions of interferon gamma and has been implicated as a factor in the onset of NEC and the progression toward fulminant disease [16]. Studies have demonstrated decreased IL-12 production in the intestinal epithelium in mice during experimental NEC [20]. This is consistent with our results, and improvement secondary to $\mathrm{H}_{2} \mathrm{~S}$ administration is a positive indicator that the pathology was resolving.

In the lung and liver tissue, similar patterns were noted in the vehicle treated NEC group compared to control with regard to IL-6 and IL-1 $\beta$. However, VEGF decreased in the vehicle group compared to control. IL-12 was not evaluated in the liver and lung because it is thought to be more of an intestinal marker for NEC [20]. Treatment with GYY4137 did result in an improvement in IL-6 and VEGF, but the rest of 
the donors did not appear to facilitate a significant improvement in the systemic inflammatory response. These data indicate that despite the improvement in intestinal injury that resulted from direct application to threatened bowel, the systemic effects of experimental NEC were likely not completely ameliorated by this therapy. Further investigation into the effects of $\mathrm{H}_{2} \mathrm{~S}$ on systemic inflammation is therefore warranted.

\section{LIMITATIONS}

Since the exact cause of NEC is not completely understood, we are limited in the types of animal models that are available for use. As such, clinical correlation to human illness may be limited. Although the clinical scores and intestinal perfusion parameters are strong indicators of the development of NEC, the diagnosis can only be established at postmortem evaluation of the intestine, and this makes it difficult to assess during the experiment whether or not NEC is being prevented. Additionally, it is difficult to blind clinical assessments of the animals, as they were performed by the same person who administered the medication. This was remedied by a very structured approach to clinical assessment, in addition to including more objective measures like intestinal perfusion and cytokine analysis. All macroscopic and histologic evaluation was blinded, and corresponded with other clinical assessments.

\section{CONCLUSION}

$\mathrm{H}_{2} \mathrm{~S}$ administration is beneficial in experimental NEC. Mouse pups treated with $\mathrm{H}_{2} \mathrm{~S}$ experienced only marginal weight gain, but did exhibit improved clinical status, increased intestinal perfusion, reduced intestinal injury, and normalization of inflammatory markers as compared to vehicle. Further experimentation into 
mechanism, mode of delivery, and adverse effects is necessary prior to widespread therapeutic use.

\section{FIGURE LEGENDS}

Figure 1: $H_{2}$ S improves weight gain and clinical status in experimental NEC. A) The vehicle group had virtually no weight gain compared to control, and though the $\mathrm{H}_{2} \mathrm{~S}$ groups had very minimal, it was statistically better than the vehicle group. B) Clinical assessment scores were higher (worse) in the vehicle group compared to control, and improved in the $\mathrm{H}_{2} \mathrm{~S}$-treated groups. Transverse bars represent median clinical score and each symbol represents an individual pup's score. ( ${ }^{*}: p<0.05$ vs. breastfed, \#: $\mathrm{p}<0.05$ vs. vehicle)

Figure 2: $\mathrm{H}_{2} \mathrm{~S}$ improves intestinal perfusion in NEC as measured by LDI compared to vehicle. A) Perfusion on P9 is reported as a percentage of baseline perfusion on P5. Perfusion was significantly lower in the vehicle group and improved in all three $\mathrm{H}_{2} \mathrm{~S}$ treated groups. ( ${ }^{*}: p<0.05$ vs. breastfed, \#: $p<0.05$ vs. vehicle) B) Representative pictures from LDI software of each group's transcutaneous intestinal perfusion. Red areas indicated higher perfusion, and blue areas indicated lower levels of perfusion. The left side of each image is the pelvis and the right is the diaphragm.

Figure 3: $\mathrm{H}_{2} \mathrm{~S}$ reduces intestinal injury in NEC compared to vehicle. A) Macroscopic injury score was higher (worse) in the vehicle group as compared to the breastfed animals. All three $\mathrm{H}_{2} \mathrm{~S}$-treated groups had improved scores. B) Histologic injury scores were higher (worse) in the vehicle group compared to the breastfed group. All three 
$\mathrm{H}_{2} \mathrm{~S}$-treated groups had improved scores. ( ${ }^{*}: p<0.05$ vs. breastfed, \#: $p<0.05$ vs. vehicle) C) Representative sections from each treatment group (H\&E stain, 20x).

Figure 4: $\mathrm{H}_{2} \mathrm{~S}$ normalizes intestinal inflammation in NEC compared to vehicle. The vehicle group had significantly decreased IL-6, IL-1 compared to control. Each $\mathrm{H}_{2} \mathrm{~S}$-treated group had levels closer to breastfed control indicating normalization in local inflammation. $\left({ }^{*}: p<0.05\right.$ vs. breastfed, $\#$ : $p<0.05$ vs. vehicle)

Figure 5: $\mathrm{H}_{2} \mathrm{~S}$ normalizes systemic inflammatory response in NEC compared to vehicle. The vehicle group had significantly decreased IL-6, VEGF, and IL-1 $\beta$ compared to control. $\mathrm{H}_{2} \mathrm{~S}$ treatment may have facilitated some normalization back to breastfed control levels, however this was not a statistically significant difference, with the exception of the GYY4137 group for IL-6 and VEGF. $\left({ }^{*}: p<0.05\right.$ vs. breastfed, $\#: p<0.05$ vs. vehicle) 


\section{REFERENCES:}

[1] Fitzgibbons SC, Ching Y, Yu D, Carpenter J, Kenny M, Weldon C, et al. Mortality of necrotizing enterocolitis expressed by birth weight categories. Journal of pediatric surgery 2009;44(6):10725; discussion 5-6.

[2] Lin HC, Wu SF, Underwood M. Necrotizing enterocolitis. The New England journal of medicine 2011;364(19):1878-9; author reply 9.

[3] Neu J, Walker WA. Necrotizing enterocolitis. The New England journal of medicine 2011;364(3):255-64.

[4] Jensen AR, Drucker NA, Khaneki S, Ferkowicz MJ, Markel TA. Hydrogen sulfide improves intestinal recovery following ischemia by endothelial nitric oxide-dependent mechanisms. Am J Physiol Gastrointest Liver Physiol 2017;312(5):G450-G6.

[5] Nino DF, Sodhi CP, Hackam DJ. Necrotizing enterocolitis: new insights into pathogenesis and mechanisms. Nature reviews Gastroenterology \& hepatology 2016;13(10):590-600.

[6] Jensen AR, Drucker NA, Khaneki S, Ferkowicz MJ, Yoder MC, DeLeon ER, et al. Hydrogen Sulfide: A Potential Novel Therapy for the Treatment of Ischemia. Shock 2017.

[7] Sodha NR, Clements RT, Feng J, Liu Y, Bianchi C, Horvath EM, et al. Hydrogen sulfide therapy attenuates the inflammatory response in a porcine model of myocardial ischemia/reperfusion injury. The Journal of thoracic and cardiovascular surgery 2009;138(4):977-84.

[8] Yin J, Tu C, Zhao J, Ou D, Chen G, Liu Y, et al. Exogenous hydrogen sulfide protects against global cerebral ischemia/reperfusion injury via its anti-oxidative, anti-inflammatory and anti-apoptotic effects in rats. Brain research 2013;1491:188-96.

[9] Gheibi S, Aboutaleb N, Khaksari M, Kalalian-Moghaddam H, Vakili A, Asadi Y, et al. Hydrogen sulfide protects the brain against ischemic reperfusion injury in a transient model of focal cerebral ischemia. Journal of molecular neuroscience : MN 2014;54(2):264-70.

[10] Li B, Zani A, Martin Z, Lee C, Zani-Ruttenstock E, Eaton S, et al. Intestinal epithelial cell injury is rescued by hydrogen sulfide. Journal of pediatric surgery 2016;51(5):775-8.

[11] Zhao Y, Biggs TD, Xian M. Hydrogen sulfide (H2S) releasing agents: chemistry and biological applications. Chemical communications 2014;50(80):11788-805.

[12] Li L, Whiteman M, Guan YY, Neo KL, Cheng Y, Lee SW, et al. Characterization of a novel, watersoluble hydrogen sulfide-releasing molecule (GYY4137): new insights into the biology of hydrogen sulfide. Circulation 2008;117(18):2351-60.

[13] Karwi QG, Bornbaum J, Boengler K, Torregrossa R, Whiteman M, Wood ME, et al. AP39, a mitochondria-targeting hydrogen sulfide $(\mathrm{H} 2 \mathrm{~S})$ donor, protects against myocardial reperfusion injury independently of salvage kinase signalling. British journal of pharmacology 2017;174(4):287-301.

[14] Zheng Y, Ji X, Ji K, Wang B. Hydrogen sulfide prodrugs-a review. Acta pharmaceutica Sinica B 2015;5(5):367-77.

[15] Chatzianastasiou A, Bibli SI, Andreadou I, Efentakis P, Kaludercic N, Wood ME, et al. Cardioprotection by H2S Donors: Nitric Oxide-Dependent and Independent Mechanisms. The Journal of pharmacology and experimental therapeutics 2016;358(3):431-40.

[16] Markel TA, Crisostomo PR, Wairiuko GM, Pitcher J, Tsai BM, Meldrum DR. Cytokines in necrotizing enterocolitis. Shock 2006;25(4):329-37.

[17] Jin X, Zimmers TA, Zhang Z, Pierce RH, Koniaris LG. Interleukin-6 is an important in vivo inhibitor of intestinal epithelial cell death in mice. Gut 2010;59(2):186-96.

[18] Sprague AH, Khalil RA. Inflammatory cytokines in vascular dysfunction and vascular disease. Biochemical pharmacology 2009;78(6):539-52. 
[19] Ferrara N, Gerber HP, LeCouter J. The biology of VEGF and its receptors. Nat Med 2003;9(6):66976.

[20] Nadler EP, Dickinson E, Knisely A, Zhang XR, Boyle P, Beer-Stolz D, et al. Expression of inducible nitric oxide synthase and interleukin-12 in experimental necrotizing enterocolitis. The Journal of surgical research 2000;92(1):71-7.

[21] Zani A, Cordischi L, Cananzi M, De Coppi P, Smith VV, Eaton S, et al. Assessment of a neonatal rat model of necrotizing enterocolitis. European journal of pediatric surgery : official journal of Austrian Association of Pediatric Surgery [et al] = Zeitschrift fur Kinderchirurgie 2008;18(6):4236.

[22] Jilling T, Simon D, Lu J, Meng FJ, Li D, Schy R, et al. The roles of bacteria and TLR4 in rat and murine models of necrotizing enterocolitis. Journal of immunology 2006;177(5):3273-82.

[23] Le Trionnaire S, Perry A, Szczesny B, Szabo C, Winyard PG, Whatmore JL, et al. The synthesis and functional evaluation of a mitochondria-targed hydrogen sulfide donor, (10-oxo-10-(4-(3-thioxo3H-1,2-dithiol-5-yl)phenoxyl)decyl)triphenylphosphonium bromide (AP39). MedChemComm 2014;5(6):728-36.

[24] Jensen AR, Manning MM, Khaneki S, Drucker NA, Markel TA. Harvest tissue source does not alter the protective power of stromal cell therapy after intestinal ischemia and reperfusion injury. The Journal of surgical research 2016;204(2):361-70.

[25] Jensen AR, Doster DL, Hunsberger EB, Manning MM, Stokes SM, Barwinska D, et al. Human Adipose Stromal Cells Increase Survival and Mesenteric Perfusion Following Intestinal Ischemia and Reperfusion Injury. Shock 2016;46(1):75-82.

[26] Pavoni V, Nicoletti P, Benemei S, Materazzi S, Perna F, Romagnoli S, et al. Effects of hydrogen sulfide (H2S) on mesenteric perfusion in experimental induced intestinal ischemia in a porcine model. Heart, lung and vessels 2015;7(3):231-7.

[27] Liu H, Bai XB, Shi S, Cao YX. Hydrogen sulfide protects from intestinal ischaemia-reperfusion injury in rats. The Journal of pharmacy and pharmacology 2009;61(2):207-12.

[28] Patel RM, Kandefer S, Walsh MC, Bell EF, Carlo WA, Laptook AR, et al. Causes and timing of death in extremely premature infants from 2000 through 2011. The New England journal of medicine 2015;372(4):331-40.

[29] Yang G, Wang R. H2S and Blood Vessels: An Overview. Handbook of experimental pharmacology 2015;230:85-110.

[30] Altaany Z, Ju Y, Yang G, Wang R. The coordination of S-sulfhydration, S-nitrosylation, and phosphorylation of endothelial nitric oxide synthase by hydrogen sulfide. Science signaling 2014;7(342):ra87.

[31] Mustafa AK, Sikka G, Gazi SK, Steppan J, Jung SM, Bhunia AK, et al. Hydrogen sulfide as endothelium-derived hyperpolarizing factor sulfhydrates potassium channels. Circulation research 2011;109(11):1259-68.

[32] Teague B, Asiedu S, Moore PK. The smooth muscle relaxant effect of hydrogen sulphide in vitro: evidence for a physiological role to control intestinal contractility. British journal of pharmacology 2002;137(2):139-45.

[33] Paul BD, Snyder SH. H2S: A Novel Gasotransmitter that Signals by Sulfhydration. Trends in biochemical sciences 2015;40(11):687-700.

[34] Wu D, Luo N, Wang L, Zhao Z, Bu H, Xu G, et al. Hydrogen sulfide ameliorates chronic renal failure in rats by inhibiting apoptosis and inflammation through ROS/MAPK and NF-kappaB signaling pathways. Scientific reports 2017;7(1):455.

[35] Jordan M, Otterness IG, Ng R, Gessner A, Rollinghoff M, Beuscher HU. Neutralization of endogenous IL- 6 suppresses induction of IL-1 receptor antagonist. Journal of immunology 1995;154(8):4081-90. 
[36] Edelson MB, Bagwell CE, Rozycki HJ. Circulating pro- and counterinflammatory cytokine levels and severity in necrotizing enterocolitis. Pediatrics 1999;103(4 Pt 1):766-71.

[37] von Boyen GB, Steinkamp M, Reinshagen M, Schafer KH, Adler G, Kirsch J. Proinflammatory cytokines increase glial fibrillary acidic protein expression in enteric glia. Gut 2004;53(2):222-8. 
Figure 1

A

Change in Weight

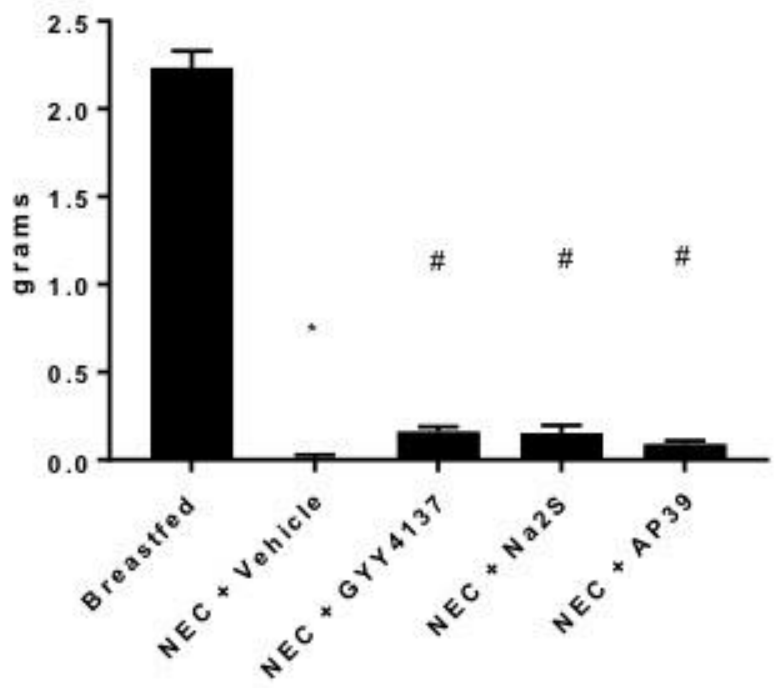

B

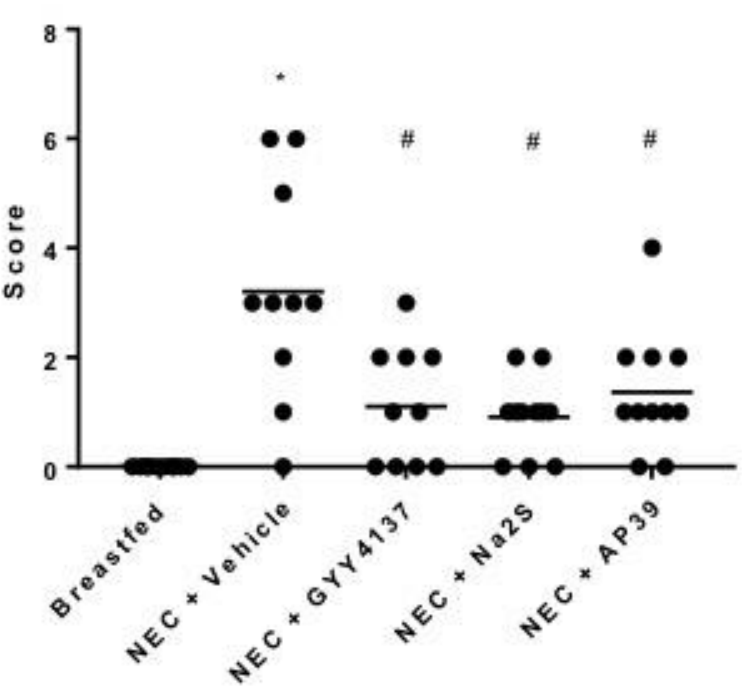


Figure 2

A

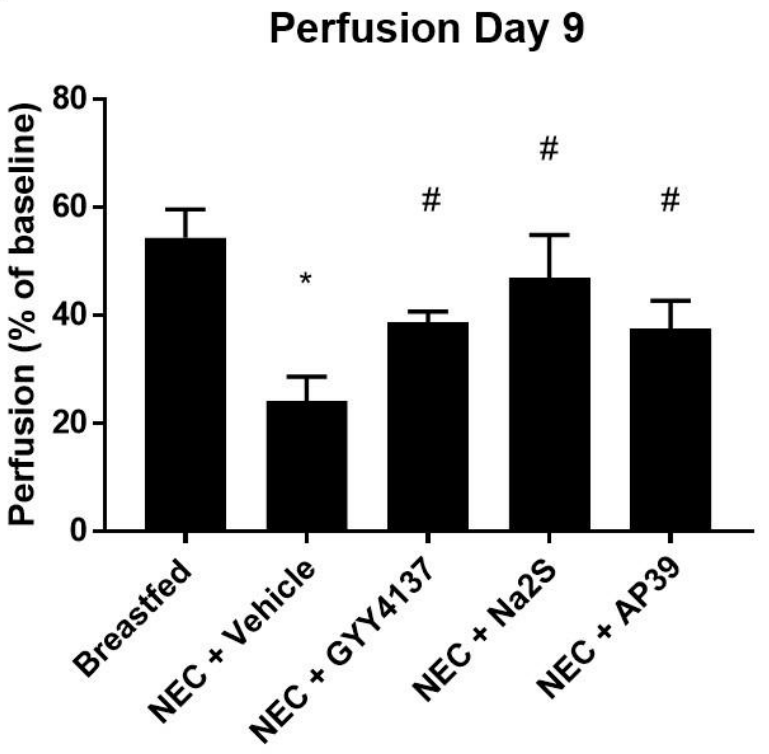

B

\section{Breastfed}

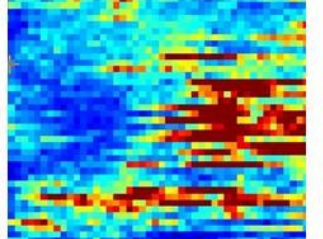

NEC + GYY4137

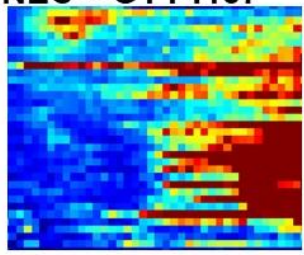

NEC + AP39

NEC + Vehicle
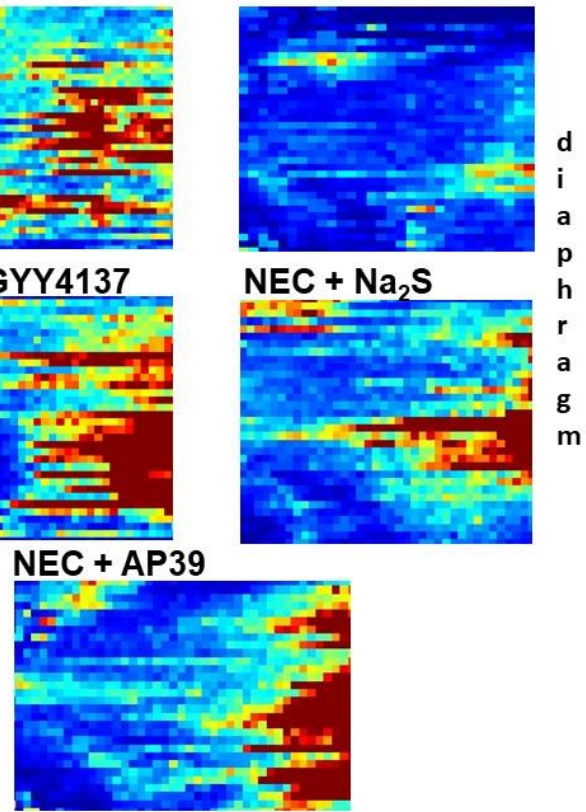
Figure 3

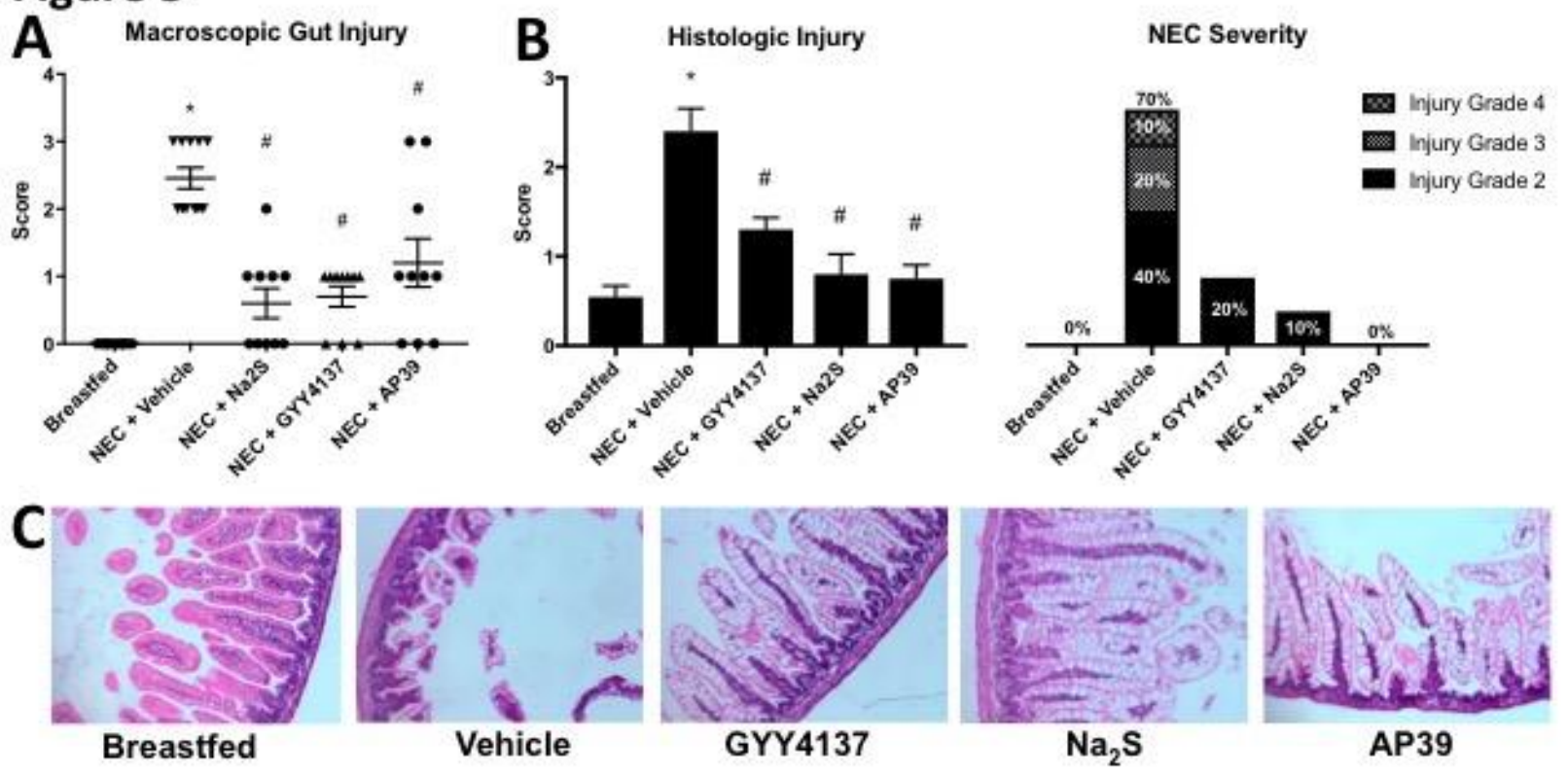


Figure 4

Intestine IL-6

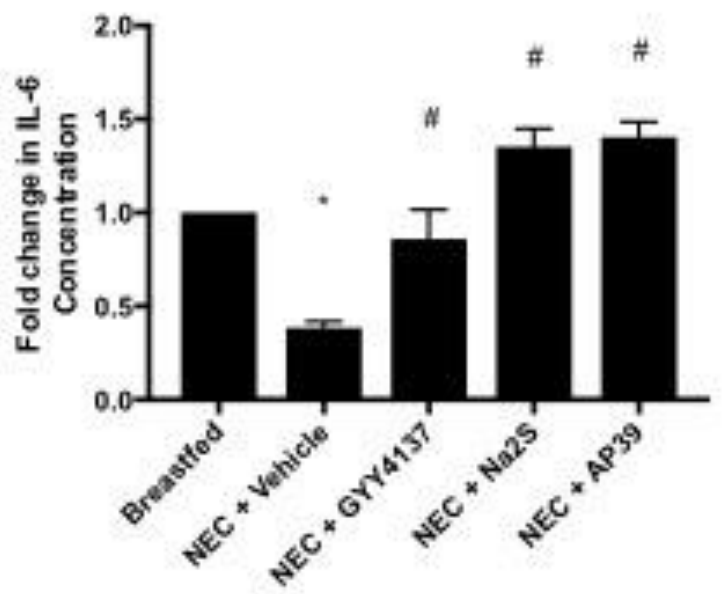

Intestine IL-1B

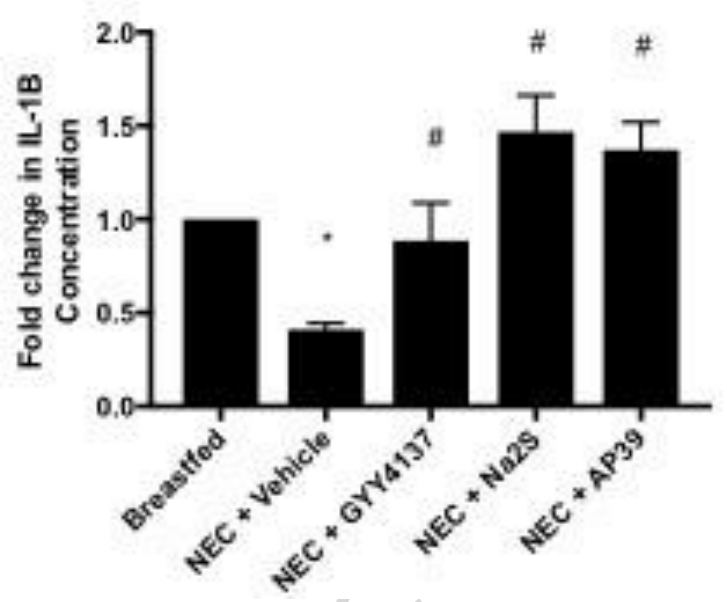

Intestine VEGF

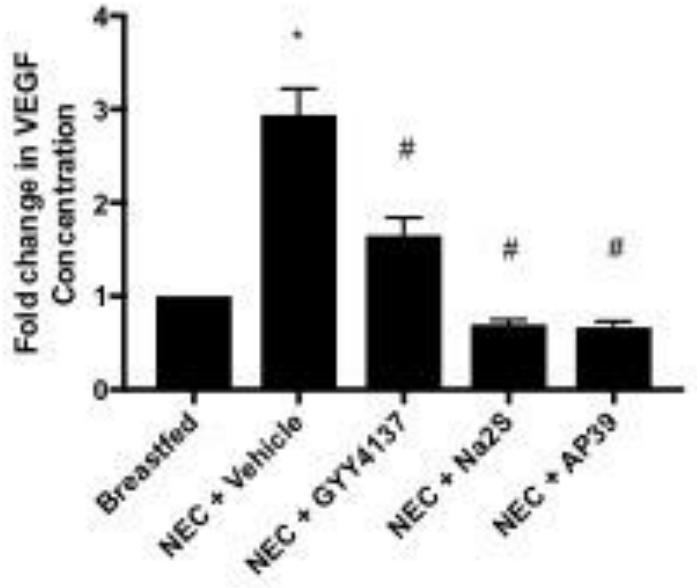

Intestine IL-12

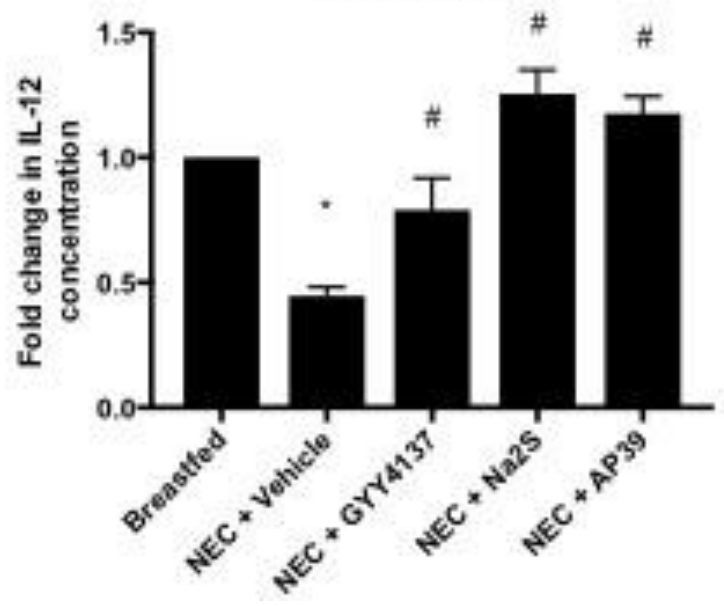


Figure 5

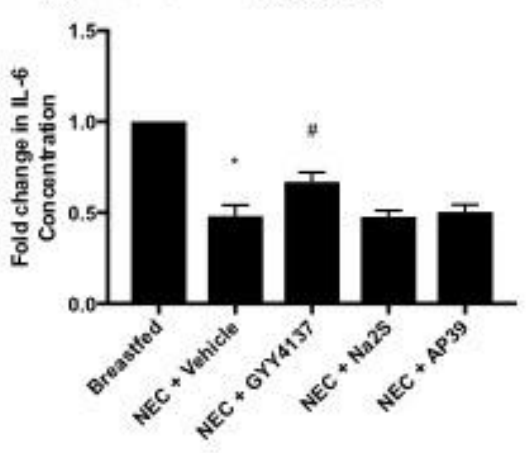

Lung IL-6

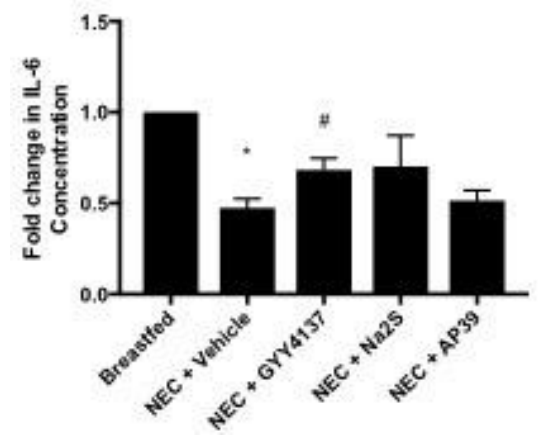

Liver VEGF

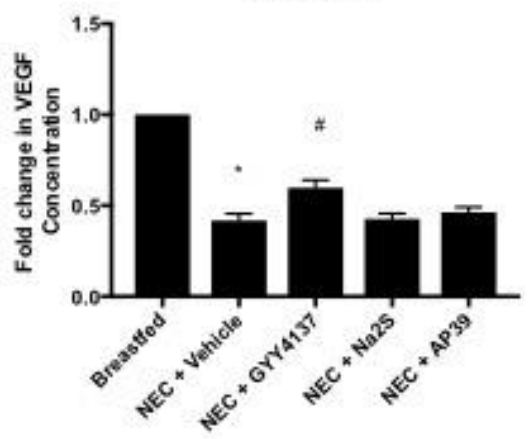

Lung VEGF

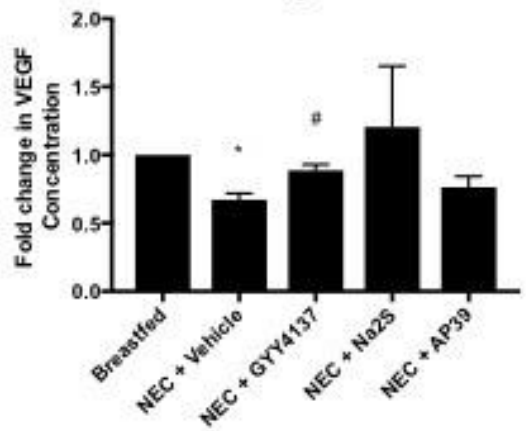

Liver IL-1B

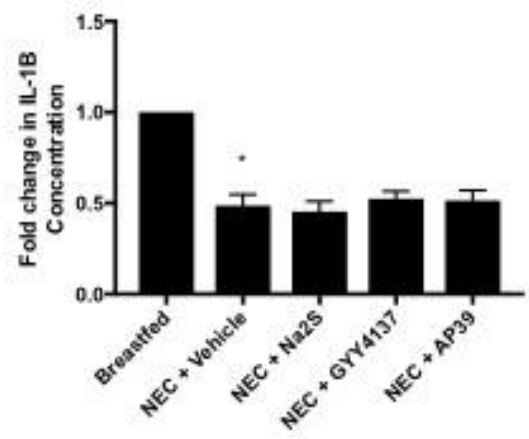

Lung IL-1B

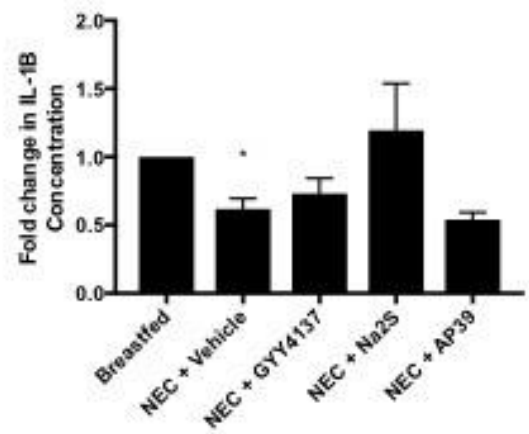




\begin{tabular}{|}
\hline \multicolumn{2}{|l|}{ Table 1. Clinical Assessment Score } \\
\hline Appearance \\
\hline 0 & Tonic and well-hydrated, glossy skin \\
\hline 1 & Slimmer, with visible skin folds \\
\hline 2 & Skinny, dehydrated \\
\hline 3 & Gasping, in agony \\
\hline Natural Activity \\
\hline 0 & Moving normally in the cage \\
\hline 1 & Wriggles if put supine \\
\hline 2 & Does not wriggle if put supine \\
\hline 3 & $\begin{array}{l}\text { Not moving limbs, lying still, supine or lateral } \\
\text { decubitus }\end{array}$ \\
\hline Response to Touch \\
\hline 0 & Alert without any stimulation \\
\hline 1 & Responding to mild stimulation (gentle foot touch) \\
\hline 2 & Responding to vigorous stimulation (foot pinch) \\
\hline 3 & $\begin{array}{l}\text { Unresponsive to vigorous stimulation (does not } \\
\text { become alert when picked up and moved) }\end{array}$ \\
\hline Body Color \\
\hline 0 & Pink \\
\hline 1 & Pale or dark distal extremities, fingers/toes \\
\hline 2 & Pale whole body \\
\hline 3 & Grey \\
\hline Zani et. al. 2008 \\
\hline
\end{tabular}

Zani et. al. 2008 


\begin{tabular}{|c|l|}
\hline \multicolumn{2}{|l|}{ Table 2. Macroscopic Gut Assessment Score } \\
\hline Consistency \\
\hline 0 & $\begin{array}{l}\text { Normal, does not tear when dissecting from } \\
\text { surrounding tissue }\end{array}$ \\
\hline 1 & Moderately friable \\
\hline 2 & Liquefied, jelly-like, extremely friable \\
\hline Color \\
\hline 0 & Normal, yellow/tan \\
\hline 1 & Patchy discoloration, pink, congested \\
\hline 2 & Extensive discoloration, dark, necrotic \\
\hline Dilation \\
\hline 0 & Normal, no dilation \\
\hline 1 & Patchy areas of dilation \\
\hline 2 & Extensive dilation throughout small bowel \\
\hline
\end{tabular}

Zani et. al. 2008 